\title{
COMMENTS
}

\section{Comment on "Effect of growth interruptions on the light emission and indium clustering of InGaN/GaN multiple quantum wells" [Appl. Phys. Lett. 79, 2594 (2001)]}

\author{
R. W. Martin, ${ }^{\text {a) }}$ K. P. O'Donnell, and P. R. Edwards \\ Department of Physics, Strathclyde University, Glasgow, G4 ONG United Kingdom
}

(Received 2 January 2002; accepted 29 August 2002)

(C) 2002 American Institute of Physics. [DOI: 10.1063/1.1515886]

The origin of the bright luminescence of nitride devices and its relation to the nanostructure of InGaN films is the subject of an intense and continuing debate. Using transmission electron microscopy (TEM) to investigate InGaN quantum wells (QWs), Chichibu et al. ${ }^{1}$ and Narukawa et al. ${ }^{2}$ were the first to argue for the existence of naturally occurring Inrich quantum dots. Energy dispersive x-ray (EDX) microanalysis on and between "dot-like" regions of high contrast gave InN mole fractions of approximately $17 \%$ and $8 \%$, respectively. ${ }^{2}$ Subsequently some of the present authors extended these ideas to argue for the formation of nearly pure InN quantum dots in InGaN QWs, with the size of the dots, rather than their composition, being primarily responsible for determining the spectral energies. ${ }^{3-5}$

In a recent letter Cho et al. ${ }^{6}$ present energy filtered transmission electron microscopy (EFTEM), which offers information on elemental composition with extremely high spatial resolution, better even than that of TEM-EDX. Their data indicate a spontaneous $\mathrm{InN}-\mathrm{GaN}$ segregation in $\mathrm{InGaN}$ quantum wells which have not experienced an interruption in growth before the top barrier layer is deposited. We wish to draw attention to the almost complete absence of an indium signal within certain interrogated regions. In particular, Fig. 4(c) of Ref. 6 shows a fluctuation between approximately $40 \%$ and $5 \% \mathrm{InN}$, with a typical length scale of $2 \mathrm{~nm}$. We will argue that this figure represents the lowest estimates for the segregation.

With any measurement of microcomposition, it is essential to consider the volume addressed in the measurement process. For an optical technique this volume is defined by the excitonic radius while in TEM-based microanalysis the investigated volume results from the spread of the electron trajectories as the beam traverses the sample, as well as the sample thickness. For the EFTEM measurements, with a beam diameter of $\sim 1 \mathrm{~nm}$ and a sample thickness in the region of $20 \mathrm{~nm}$, the measured $\mathrm{InN}$ mole fractions therefore correspond to values averaged within a volume of tens of cubic nanometers. For their EDX experiments, Narukawa et al. used a $100 \mathrm{kV}$ beam with a $1 \mathrm{~nm}$ diameter spot. MonteCarlo simulations indicate that such a beam will spread to a

a)Electronic mail: r.w.martin@strath.ac.uk diameter of approximately $3 \mathrm{~nm}$ while traversing a specimen of thickness about $30 \mathrm{~nm}$.

In the EFTEM traverse across the QWs grown without interruption, the peaks of $40 \% \mathrm{InN}$ content represent an average over the excitation cone and similar fluctuations would be expected to occur along the direction of the incident beam (perpendicular to the traverse and also in the plane of the quantum well). The most likely nanostructure thus appears to be a set of In-rich quantum dots, about $2 \mathrm{~nm}$ in diameter, that occupy only a small fraction of the total volume. To reach an average In content of $40 \%$ in any of the excitation cones requires a much higher local $\mathrm{InN}$ content within the dots, probably approaching $100 \%$. The data cannot distinguish a $\mathrm{GaN}$ matrix containing nearly pure $\mathrm{InN}$ dots occupying $40 \%$ of this volume from a column with average composition $\mathrm{In}_{0.4} \mathrm{Ga}_{0.6} \mathrm{~N}$, but the equivalence of the two directions in the plane of the quantum well point to the former. The minima are even more thought provoking, given that they indicate an almost complete absence of indium nitride within a volume of tens of cubic nanometers in material where the overall mean InN content can be estimated at $\sim 17 \%$ [by averaging the entire trace in Fig. 4(c) of Ref. 6]. It is worth pointing out that the globally averaged In content in the wells $(\sim 17 \%)$ agrees well with the observed photoluminescence emission energy $\left(E_{p}\right)$ of $2.8 \mathrm{eV}$, according to our published expression for $E_{p}(x) .{ }^{7}$ Such a situation cannot be explained by anything other than extreme InN-GaN segregation. $\{\mathrm{We}$ are, however, unable to suggest an explanation at present for the apparent increase in mean indium fraction in the QWs subject to a growth delay or for the absence of a shift in $E_{p}$ between QWs with differing average indium contents of $24 \%$ and $31 \%$ [Fig. 4(d) of Ref. 6].\}

Such considerations lead to an important re-evaluation of the data presented in Ref. 2. Here the areal density of the In-rich dot-like regions is estimated to be $5 \times 10^{11} \mathrm{~cm}^{-2}$, giving a volume filling factor of $2 \%$ for the dots. An average dot diameter of $3 \mathrm{~nm}$ corresponds to about ten dots per well in the $100 \mathrm{~nm}$ by $30 \mathrm{~nm}$ area illustrated in Fig. 2(b) of Ref. 2. Following the author's assumption of no overlapping of dots, the EDX probe beam will encounter at most one dot in its $30 \mathrm{~nm}$ path, for which the measured In-content is only an average. The local In content in the isolated dots therefore 


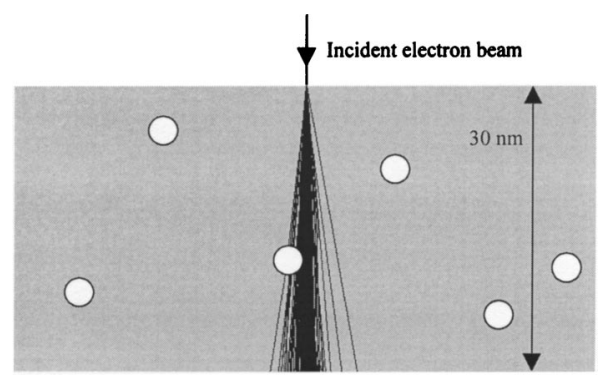

FIG. 1. Schematic representation of the measurement in Ref. 2 using a Monte-Carlo simulation of the trajectories of one hundred thousand 100 $\mathrm{keV}$ electrons. Nearly pure $\mathrm{InN}$ dots, $3 \mathrm{~nm}$ in diameter and with areal density $5 \times 10^{11} \mathrm{~cm}^{-2}$, are represented by the white dots. The shaded region represents an area $30 \times 60 \mathrm{~nm}$.

approaches $100 \%$. Figure 1 illustrates the situation, using Monte-Carlo simulations of the trajectory of 100000 electrons in the incident beam. As mentioned by the authors of Ref. 2, measuring the residual In content in the barriers will be complicated by the spread of the electron beam into neighboring wells.

In conclusion, we suggest that the TEM microanalyses, presented recently by Cho et al. and almost 5 years ago by Narukawa et al., appear to support the idea of an extreme
InN-GaN segregation in InGaN heterostructures, which results in the spontaneous formation of nearly pure InN quantum dots. In both cases the reported In contents have been drastically reduced by the volume averaging that is inherent in such measurements. In all techniques that probe the nanostructure of thin films we stress the importance of considering the measured volume. To directly probe the local structure of an $\mathrm{InN}$ quantum dot requires a technique that is capable of addressing only a few cubic nanometers of material.

${ }^{1}$ S. Chichibu, T. Azuhata, T. Sota, and S. Nakamura, Appl. Phys. Lett. 69, 4188 (1996).

${ }^{2}$ Y. Narukawa, Y. Kawakami, M. Funato, S. Fujita, and S. Nakamura, Appl. Phys. Lett. 70, 981 (1997).

${ }^{3}$ K. P. O'Donnell, R. W. Martin, and P. G. Middleton, Phys. Rev. Lett. 82, 237 (1999).

${ }^{4}$ R. W. Martin and K. P. O’Donnell, Phys. Status Solidi B 216, 441 (1999).

${ }^{5}$ L. Nistor, H. Bender, A. Vantomme, M. F. Wu, J. Van Landuyt, K. P. O'Donnell, R. Martin, K. Jacobs, and I. Moerman, Appl. Phys. Lett. 79, 2594 (2001)

${ }^{6}$ H. K. Cho, J. Y. Lee, N. Sharma, C. J. Humphreys, G. M. Yang, C. S. Kim, J. H. Song, and P. W. Yu, Appl. Phys. Lett. 79, 2594 (2001).

${ }^{7}$ K. P. O'Donnell, R. W. Martin, C. Trager-Cowan, M. E. White, K. Esona, C. Deatcher, P. G. Middleton, K. Jacobs, W. Van der Stricht, C. Merlet, B. Gil, A. Vantomme, and J. F. W. Mosselmans, Mater. Sci. Eng., B 82, 194 (2001). 